\title{
Surgical Simulation of Endoscopic Sinus Surgery using Thiel Soft-Embalmed Human Cadaver
}

\author{
Precious Eunice R. Grullo, ${ }^{1}$ Harivelle Charmaine T. Hernando, ${ }^{2}$ Ryner Jose DC. Carrillo, ${ }^{1,2}$ Pio Renato F. Villacorta, ${ }^{2}$ \\ Josefino G. Hernandez, ${ }^{1}$ Ramon Antonio B. Lopa, ${ }^{1}$ Arsenio Claro A. Cabungcal ${ }^{1}$ and Jupiter Kelly H. Barroa ${ }^{2}$ \\ ${ }^{1}$ Department of Otorhinolaryngology, College of Medicine and Philippine General Hospital, University of the Philippines Manila \\ ${ }^{2}$ Department of Anatomy, College of Medicine, University of the Philippines Manila
}

\begin{abstract}
Objective. This study describes the quality of Thiel softembalmed cadavers as training model for endoscopic sinus surgery in terms of color and consistency of the tissues and similarity of performing the surgical steps to live surgery.

Methods. This is a cross-sectional descriptive study. Six Thiel softembalmed cadavers from the University of the Philippines, College of Medicine, Department of Anatomy were used as training models. The Thiel-preserved cadavers utilized the soft embalming protocol being employed at the Virginia State Anatomical Program in Richmond, Virginia, USA. Ten otorhinolaryngologists were recruited to evaluate the cadavers using a questionnaire with three parts. The first two parts utilize a 10-point Likert scale with 1 as the least similar to live patient while 10 as simulating the live patient. The third part is an openended question regarding the suitability of Thiel soft-embalmed cadavers in the training for endoscopic sinus surgery.
\end{abstract}

Results. Endoscopic sinus surgery was successfully performed in all cadavers. The Thiel soft-embalmed cadaver closely replicates the color and consistency of the anatomic structures important in endoscopic sinus surgery. All the surgical steps were performed with ease simulating live surgery.

Conclusion. Thiel soft-embalmed cadaver is a suitable model for training in endoscopic sinus surgery.

Key Words: Thiel cadaver, endoscopic sinus surgery, surgical model

\section{Introduction}

Complications arising from Endoscopic Sinus Surgery (ESS) vary from minor ones including middle turbinate adhesions and bleeding to major complications like orbital injury, cerebrospinal fluid leaks and death. The incidence was reported to range from 1.0 to $22.9 \% .^{1-3}$ To ensure patient safety, otorhinolaryngologists should be adequately trained

Corresponding author: Precious Eunice R. Grullo MD, MPH

Department of Otorhinolaryngology

2nd Floor, Ward 10

Philippine General Hospital

University of the Philippines Manila

Taft Avenue, Ermita, Manila 1000 Philippines

Telephone: +6325548400 local 2151 or 2152

Email: prgrullo@up.edu.ph before doing this surgical procedure on live patients. Courses on endoscopic sinus surgery are a prerequisite for clinical practice.

Training courses on this surgical procedure have stations with a surgical model, a set of endoscopes and sinus surgery instruments. ${ }^{4}$ Surgical models include anatomic models, animals like sheep, virtual simulators and cadavers in order to teach the anatomy and to allow the trainees to practice endoscopic surgical skills in a bloodless surgical field. ${ }^{4-7}$ Anatomic models and formalin-embalmed cadavers resembled the anatomy of the human body; however, anatomic models and formalin-embalmed cadavers have poor soft tissue flexibility, poor pliability and stiff joints. Formalinembalmed cadavers also have poorly preserved surgical planes, tissue texture and color. Thus, fresh frozen cadavers are considered the gold standard in academic and training purposes. Fresh frozen cadavers mimic normal human tissue consistency and joint movement, thus simulating live patients. However, these are very expensive, can be used for a short duration of time and have biosafety issues. ${ }^{8}$

Introduced in 1992, Thiel soft-embalmed cadavers have been increasingly utilized in research and training in the past two decades. This alternative preservation method results in cadavers with color and soft tissue elasticity values similar to live human soft tissue. ${ }^{9}$ In a systematic review by Gilbody et al., they pointed that experienced surgeons consider Thiel soft-embalmed cadavers to closely mimic live surgeries..$^{10}$ Several publications have reported the use of Thiel cadavers in simulating live surgical and diagnostic procedures for training and research. ${ }^{11-19}$

This study aims to describe the quality of Thiel softembalmed cadavers as a training model for endoscopic sinus surgery in terms of color and consistency of the tissues and similarity of performing the surgical steps to live surgery. Based on the results of this study, the Thiel soft-embalmed cadaver could be a suitable model for academic and training purposes.

\section{Materials and Methods}

This study employed a cross-sectional descriptive study design. Six Thiel soft-embalmed cadavers from the University of the Philippines, College of Medicine, Department of Anatomy were used as training models. The 
Thiel-preserved cadavers utilized the soft embalming protocol being employed at the Virginia State Anatomical Program in Richmond, Virginia, USA. The process consists of a pre-injection solution and an arterial injection solution utilizing two separately formulated solutions (A and B) that are later combined with additional chemicals.

The pre-injection solution utilized is a chemical called META-FLOW (manufactured by Dodge Chemical Company) consisting of one 16-ounce bottle mixed with a half gallon of water.

The chemicals used for Solution A are: Boric Acid, Monoethylene glycol, ammonium nitrate and potassium nitrate; for Solution B: mono-ethylene glycol and 4-chloro-3methylphenol. Both solutions are mixed together with sodium sulphite, morpholine, formalin and denatured ethyl alcohol. This final solution constitutes the arterial injection solution.

Ten otorhinolaryngologists who have performed endoscopic sinus surgery more than five times were recruited as study participants. Three of the participants were consultants who have performed anterior skull base surgeries and endoscopic sinus surgeries for more than 10 years. The remaining 7 participants were senior residents. This study was submitted and approved by the University of the Philippines Manila Research Ethics Board (UPMREB) Panel for ethics review. A written informed consent approved by the UPMREB Panel was secured from all participants. Each participant performed endoscopic sinus surgery using Thiel soft-embalmed cadaver. A questionnaire was given to them at the end of the procedure. The questionnaire may be answered in 10 minutes and has three parts. The first part evaluated the tissue quality of the Thiel soft-embalmed cadaver in terms of color and consistency. The second part evaluated the similarity of doing the steps in endoscopic sinus surgery using the Thiel soft-embalmed cadaver compared to their experience with a live patient. Both parts utilize a 10-point Likert scale with 1 as the least similar to a live patient while 10 as simulating a live patient. The third part is an open-ended question regarding the suitability of Thiel soft-embalmed cadavers in the training for endoscopic sinus surgery. For the first and second parts, the median of their scores are tabulated, while their responses and comments for the $3^{\text {rd }}$ part are discussed.

\section{Results}

Tables 1 and 2 show the median scores of the participants evaluating the quality of the important anatomic structures and the simulation of surgical steps in endoscopic sinus surgery.

In general, the smell of the Thiel soft-embalmed cadavers was described by some participants as fishy but tolerated by most of the participants. The outward appearance of the Thiel soft-embalmed cadavers showed a pale skin color. The eyes had lost its volume and was hypotonic compared to live patients.
Table 1. Quality of Tissues involved in Endoscopic Sinus Surgery

\begin{tabular}{lcc}
\hline Tissue & Color (median) & Consistency (median) \\
\hline Nasal ala & 8 & 8 \\
Septum & 8 & 7 \\
Turbinate & 8 & 8 \\
Uncinate Process & 8 & 8 \\
Ethmoid bulla & 8 & 8 \\
Anterior ethmoid air cells & 8 & 8 \\
Posterior ethmoid air cells & 8 & 8 \\
Sphenoid sinus & 8 & 8 \\
Frontal recess & 8 & 8 \\
Lamina papyracea & 8 & 8 \\
Sphenopalatine artery/ & 7 & 8 \\
Blood vessels & & \\
\hline
\end{tabular}

Table 2. Assessment of the Simulation of Surgical Steps in Endoscopic Sinus Surgery

\begin{tabular}{lc}
\hline Sugical steps & Median Score \\
\hline Development of mucoperichondrial flap & 8 \\
Resection of septal cartilage & 8 \\
Medialization of middle turbinate & 8 \\
Uncinectomy & 8 \\
Maxillary antrostomy & 8 \\
Removal of ethmoid bulla & 8 \\
Partial turbinectomy & 9 \\
Sphenopalatine artery ligation & 8 \\
\hline
\end{tabular}

Compared to live surgery: 1) the ala was pliable and can easily be manipulated; 2) the vibrissae were still present; 3) the nasoseptal mucosa was pink, smooth and more slimy; 4) the septal mucosa was more adherent to the cartilage but could easily be incised without exerting much pressure; 5) the mucoperichondrial flap was slightly difficult to raise; 6) medialization of the middle turbinate was reported by one participant to be more difficult; 7) the consistency of the uncinate process, ethmoid bulla, anterior and posterior ethmoid air cells were similar; 8) the performance of uncinectomy, maxillary antrostomy and ethmoidectomy were similar; 9) orbital ballottement produced less visible movement of the lamina papyracea; 10) when the lamina papyracea was intentionally entered during the dissection, the orbital fat did not come out easily; 11) identification of the sphenopalatine artery, anterior and posterior ethmoid arteries were feasible; and 12) nasoseptal flaps were easily raised by some of the participants.

Figure 1 shows the surgical steps in endoscopic sinus surgery that were done in the Thiel soft-embalmed cadaver.

As a whole, endoscopic sinus surgery was successfully performed in all the Thiel soft-embalmed cadavers.

\section{Discussion}

Thiel soft-embalmed cadavers have been used in several surgical training centers in Europe and USA, including otorhinolaryngologic procedures. The degree of suitability of Thiel soft-embalmed cadavers for training differs per surgical procedure. ${ }^{11-18}$ 

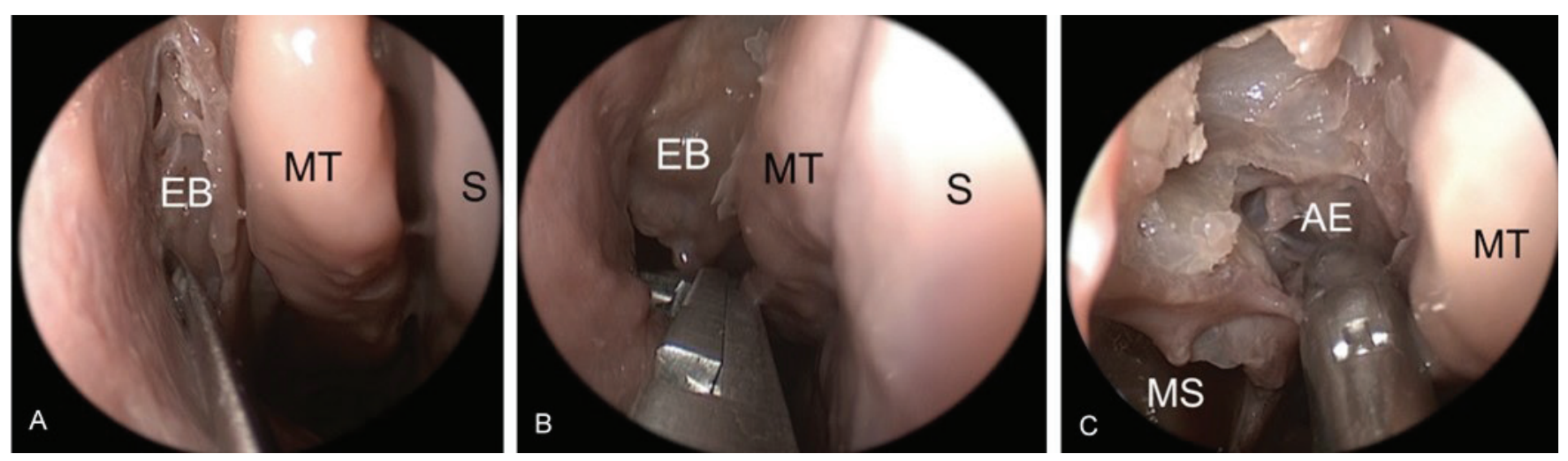

Figure 1. Surgical steps in Endoscopic Sinus Surgery as performed in the Thiel soft-embalmed cadavers. A. Uncinectomy, B. Maxillary antrostomy, C. Ethmoidectomy. (EB - ethmoid bulla; MT - middle turbinate; S - septum; AE - anterior ethmoid cells; MS - maxillary sinus mucosa)

Thiel soft-embalmed cadavers are suitable models for surgical training in endoscopic sinus surgery particularly for Residents in Training in Otorhinolaryngology. The high median scores for the consistency and color of the tissues show the appropriateness of the Thiel soft-embalmed cadavers for teaching surgical anatomy for endoscopic sinus surgery. Such should be mastered by all otorhinoloaryngologists prior to doing the actual procedure.

The high median scores for the simulation of the surgical steps in endoscopic sinus surgery demonstrate that Thiel soft-embalmed cadavers have high "task fidelity" defined as how much the trained surgical procedure resembles live surgery. ${ }^{8}$

The sphenopalatine artery, anterior and posterior ethmoid arteries were similar to live patients. The Thiel solution perfectly preserved the blood vessels leading to intact lumen of both arteries and veins resulting to blood vessels that can be dissected like live surgery. ${ }^{8}$

Thiel soft-embalmed cadavers have been recommended as good surgical models for other procedures as well. These cadavers have supple skin and subcutaneous tissues simulating live surgery in flap raising and retraction. ${ }^{11}$ The flexibility of the soft tissues makes the Thiel-embalmed cadavers inflatable thus appropriate for simulation of laparoscopic surgeries. ${ }^{12}$

In the head and neck area, Thiel embalming technique had been used in Germany for training courses in oral surgery and implantology. These training courses include surgical procedures like teeth osteotomies, soft tissue management, closing of oro-antral fistula, alveolotomies, harvesting bone grafts, sinus floor elevation, neurolysis of mental nerve and lateralization of inferior alveolar nerve. ${ }^{13}$ In a systematic review by Gilbody et al., they describe Thiel soft-embalmed cadavers look more similar to live patients than formalin-embalmed cadavers in terms of joint flexibility and tissue planes making it useful for thyroid surgery and airway procedures. ${ }^{10}$ Eisma et al. reported that Thiel soft-embalmed cadavers are better than formalin- embalmed cadavers for thyroid surgery training. ${ }^{14}$ In another study on the suitability of Thiel soft-embalmed cadavers on training, Feigl et al. concluded that Thiel softembalmed cadavers have comparable texture, color and consistency to live human tissues and are suitable models for cricothyrotomy and flexible bronchoscopy. ${ }^{15}$

Not all reports favor the use of Thiel soft-embalmed cadavers. There were reports about the soft consistency of cartilage, less compact nerves and weaker musculature. ${ }^{11}$ Fessel et al. still recommended fresh frozen cadaver in biomechanical study of tendons. In their study, it was observed that Thiel soft-embalmed cadavers have decreased elastic modiolus and different failure characteristics compared to fresh frozen cadavers. ${ }^{16}$

In this study, Thiel soft-embalmed cadavers differ from live patients in several aspects. First, the elevation of nasoseptal and mucoperichondrial flap is more difficult as the soft tissue is more adherent to the cartilage. However, this observation could be affected by the lack of hydro dissection, as local anesthesia was not injected during the dissection. Second, the mucosa is slimier than that of a live patient. This sticky Thiel solution can be washed with water resulting in a mucosa that closely resembles that of a live patient. Third, medialization of the middle turbinate was also reported to be more difficult due to the stiffness of the middle concha. This is contrary to that reported by Wolff et al. wherein cartilage become softer in Thiel soft-embalmed cadavers compared to live surgeries. ${ }^{11}$ Fourth, because of the hypotonic eyes, gentle ballottement of the globe produce less noticeable transmitted movement by the orbital contents in the operative field.

The Thiel solution was made to preserve the texture, color and flexibility of the body to as close as possible to that of a live person. Benkhadra et al. hypothesized that boric acid is the component of the Thiel solution that corrodes the proteins in the cadaver. ${ }^{20}$ In their histopathologic study, the collagen sheath remained intact and the collagen structure is preserved but the muscle fibers looked denatured. This 
resulted to a preserved form of the muscle. Gilbody et al. pointed that the high salt concentration of the Thiel solution resulted to protein denaturation causing homogenization of the tissues. ${ }^{10}$

The limitations of this study include the following: First, there were only ten participants who did the Endoscopic sinus surgery simulation. These were consultants who have more than 10 years of practice and Senior Residents in training at the University of the Philippines-Philippine General Hospital. Second, there were minimal intercadaveric differences noted in terms of tissue consistency. This variation could be due to any of the following factors: 1 ) varying periods between death and embalming; 2) differences in the vascular properties of the cadaver; 3) deficiencies in the embalming properties of the Thiel fluid; 4) age; 5) pre-cadaveric health status; 6) amount of body fat; 7) initial condition of the muscles; and 8) pathologies of other organs, affecting the absorption of the Thiel solution.

In conclusion, the Thiel soft-embalmed cadaver closely resembles a live patient in terms of anatomic structures and is similar to the quality of human tissue in appearance and consistency. The steps in endoscopic sinus surgery were simulated with high fidelity and the technical challenges in instrumentation were similar to live surgeries. Thus, the Thiel soft-embalmed cadaver is a suitable model for training in endoscopic sinus surgery.

\section{References}

1. Ramakrishnan VR, Kingdom TT, Nayak JV, Hwang PH, Orlandi RR. Nationwide incidence of major complications in endoscopic sinus surgery. Int Forum Allergy Rhinol. 2012; 2(1):34-9. doi:10.1002/alr.20101.

2. Stankiewicz JA, Lal D, Connor M, Welch K. Complications in endoscopic sinus surgery for chronic rhinosinusitis: A 25-year experience. Laryngoscope. 2011; 121(12):2684-701. doi:10.1002/lary.21446.

3. Sgambatti Celis L ${ }^{1}$, Gil Melcón M, Franco Calvo F, de la Fuente Cañibano R, del Rey Tomás-Biosca F, Batuecas Caletrio A. Complications of endoscopic sinus surgery in a residency training program. Acta Otorrinolaringol Esp. 2010; 61(5):345-50.

4. Zuckerman JD, Wise SK, Rogers GA, Senior BA, Schlosser RJ, Del Gaudio JM. The utility of cadaver dissection in endoscopic sinus surgery training courses. Am J Rhinol Allergy. 2009; 23(2):218-24. doi:10.2500/ajra.2009.23.3297.
5. Acar B, Gunbey E, Babademez MA, Karabulut H, Gunbey HP, Karasen RM. Utilization and dissection for endoscopic sinus surgery training in the residency program. J Craniofac Surg. 2010; 21(6):1715-8.

6. Burge SD, Bunegin L, Weitzel EK, McMains KC. The validation of an endoscopic sinus surgery skills training model: A pilot study. Am J Rhinol Allergy. 2012; 26(5):409-13. doi:10.2500/ajra.2012.26.3798.

7. Fortes B, Balsalobre L, Weber R, et al. Endoscopic sinus surgery dissection courses using a real simulator: the benefits of this training. Braz J Otorhinolaryngol. 2016; 82(1):26-32. doi:10.1016/j.bjorl.2015.02.003.

8. Eisma R, Wilkinson T. From "silent teachers" to models. PLoS Biol. 2014; 12(10):e1001971. doi:10.1371/journal.pbio.1001971.

9. Sangchay N. The soft cadaver (Thiel's method): The new type of cadaver of Department of Anatomy, Siriraj Hospital. Siriraj Med J. 2014; 66(Suppl):S228-31.

10. Gilbody J, Prasthofer AW, Ho K, Costa ML. The use and effectiveness of cadaveric workshops in higher surgical training: a systematic review. Ann R Coll Surg Engl. 2011; 93(5):347-52. doi:10.1308/147870811X582954.

11. Wolff K, Kesting M, Mucke T, Rau A, Holzle F. Thiel embalming technique: A valuable method for microvascular exercise and teaching of flap raising. Microsurgery. 2008; 28(4):273-8.

12. Giger U, Frésard I, Häfliger A, Bergmann M, Krähenbühl L. Laparoscopic training on Thiel human cadavers: a model to teach advanced laparoscopic procedures. Surg Endosc. 2008; 22(4):901-6.

13. Holzle F, Franz EP, Lehmbrock J, et al. Thiel embalming technique: A valuable method for teaching oral surgery and implantology. Clin Implant Dent Relat Res. 2012; 14(1):121-6.

14. Eisma R, Mahendran S, Majumdar S, Smith D, Soames RW. A comparison of Thiel and formalin embalmed cadavers for thyroid surgery training. Surgeon. 2011; 9(3):142-6. doi:10.1016/j.surge.2010.09.001.

15. Feigl G, Benkhadra M, Lenfant F, et al. Bronchoscopy and cricothyrotomy: Results from cadavers embalmed with Thiel's method compared to other embalming methods and living subjects. Acta Medico-Biotechnica. 2008; 1:25-36.

16. Fessel G, Frey K, Schweizer A, Calcagni M, Ullrich O, Snedeker JG. Suitability of Thiel embalmed tendons for biomechanical investigation. Ann Anat. 2011; 193(3):237-41. doi:10.1016/j.aanat.2011.03.007.

17. Eljamel S, Volovick A, Saliev T, Eisma R, Melzer A. Evaluation of Thiel cadaveric model for MRI-guided stereotactic procedures in neurosurgery. Surg Neurol Int. 2014; 5(Suppl 8):S404-9. doi:10.4103/ 2152-7806.140199.

18. Healy SE, Rai BP, Biyani CS, Eisma R, Soames RW, Nabi G. Thiel embalming method for cadaver preservation: A review of new training model for urologic skills training. Urology. 2015; 85(3):499-504. doi:10.1016/j.urology.2014.11.009.

19. Joy J, McLeod G, Lee N, et al. Quantitative assessment of Thiel softembalmed human cadavers using shear wave elastography. Ann Anat. 2015; 202:52-6. doi:10.1016/j.aanat.2015.06.007.

20. Benkhadra M, Bouchot A, Gerard J, et al. Flexibility of Thiel's embalmed cadavers: the explanation is probably in the muscles. Surg Radiol Anat. $2011 ; 33(4): 365-8$. 